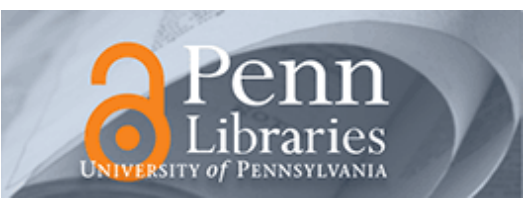

University of Pennsylvania

ScholarlyCommons

\title{
$5-4-2011$
}

\section{Lithium Fiber Growth on the Anode in a Nanowire Lithium Ion Battery During Charging}

Xiau Hua Liu

Sandia National Laboratories

Li Zhong

University of Pittsburgh - Main Campus

Li Qiang Zhang

University of Pittsburgh; Zhejiang University

Akihiro Kushima

University of Pennsylvania, kushima@seas.upenn.edu

Scott X. Mao

University of Pittsburgh - Main Campus

Follow this and additional works at: https://repository.upenn.edu/mse_papers

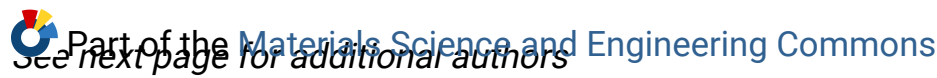

\section{Recommended Citation}

Liu, X., Zhong, L., Zhang, L., Kushima, A., Mao, S. X., Li, J., Ye, Z., Sullivan, J. P., \& Huang, J. (2011). Lithium Fiber Growth on the Anode in a Nanowire Lithium Ion Battery During Charging. Retrieved from

https://repository.upenn.edu/mse_papers/204

Suggested Citation:

X.H. Liu, et al. Lithium fiber growth on the anode in a nanowire lithium ion battery during charging. Applied Physics Letters 98, 183107.

(C) 2011 American Institute of Physics. This article may be downloaded for personal use only. Any other use requires prior permission of the author and the American Institute of Physics. The following article appeared in Applied Physics Letters and may be found at http://dx.doi.org/10.1063/1.3585655

This paper is posted at ScholarlyCommons. https://repository.upenn.edu/mse_papers/204

For more information, please contact repository@pobox.upenn.edu. 


\title{
Lithium Fiber Growth on the Anode in a Nanowire Lithium Ion Battery During Charging
}

\author{
Abstract \\ Lithium ( $\mathrm{Li}$ ) dendrite formation has been recognized as one of the major safety concerns for Li metal \\ batteries but not for conventional Li ion batteries (LIBs) where Li metal is not used. With the advanced in \\ situ transmission electron microscopy enabling direct observation of battery operation, we found that $\mathrm{Li}$ \\ fibers with length up to $35 \mu \mathrm{m}$ grew on nanowire tip after charging. The Li fibers growth were highly \\ directional, i.e., nucleating from the nanowire tip, and extending along the nanowire axis, which was \\ attributed to the strong electric field enhancement effect induced by the sharp nanowire tip. This study \\ reveals a potential safety concern of short-circuit failure for LIBs using nanowire anodes.

\section{Disciplines} \\ Engineering | Materials Science and Engineering

\section{Comments} \\ Suggested Citation: \\ X.H. Liu, et al. Lithium fiber growth on the anode in a nanowire lithium ion battery during charging. Applied \\ Physics Letters 98, 183107. \\ (C) 2011 American Institute of Physics. This article may be downloaded for personal use only. Any other \\ use requires prior permission of the author and the American Institute of Physics. The following article \\ appeared in Applied Physics Letters and may be found at http://dx.doi.org/10.1063/1.3585655

\section{Author(s)} \\ Xiau Hua Liu, Li Zhong, Li Qiang Zhang, Akihiro Kushima, Scott X. Mao, Ju Li, Zhi Zhen Ye, John P. Sullivan, \\ and Jian Yu Huang
}




\title{
Lithium fiber growth on the anode in a nanowire lithium ion battery during charging
}

\author{
Xiao Hua Liu, ${ }^{1}$ Li Zhong, ${ }^{2}$ Li Qiang Zhang, ${ }^{2,4}$ Akihiro Kushima, ${ }^{3}$ Scott X. Mao, ${ }^{2} \mathrm{Ju} \mathrm{Li},{ }^{3}$ \\ Zhi Zhen Ye, ${ }^{4}$ John P. Sullivan, ${ }^{1}$ and Jian Yu Huang ${ }^{1, a)}$ \\ ${ }^{1}$ Center for Integrated Nanotechnologies, Sandia National Laboratories, Albuquerque, \\ New Mexico 87185, USA \\ ${ }^{2}$ Department of Mechanical Engineering and Materials Science, University of Pittsburgh, Pittsburgh, \\ Pennsylvania 15261, USA \\ ${ }^{3}$ Department of Materials Science and Engineering, University of Pennsylvania, Philadelphia, \\ Pennsylvania 19104, USA \\ ${ }^{4}$ Department of Materials Science and Engineering, State Key Laboratory of Silicon Materials, \\ Zhejiang University, Hangzhou, 310027, People's Republic of China
}

(Received 14 March 2011; accepted 6 April 2011; published online 4 May 2011)

\begin{abstract}
Lithium ( $\mathrm{Li}$ ) dendrite formation has been recognized as one of the major safety concerns for $\mathrm{Li}$ metal batteries but not for conventional Li ion batteries (LIBs) where Li metal is not used. With the advanced in situ transmission electron microscopy enabling direct observation of battery operation, we found that Li fibers with length up to $35 \mu \mathrm{m}$ grew on nanowire tip after charging. The Li fibers growth were highly directional, i.e., nucleating from the nanowire tip, and extending along the nanowire axis, which was attributed to the strong electric field enhancement effect induced by the sharp nanowire tip. This study reveals a potential safety concern of short-circuit failure for LIBs using nanowire anodes. (C) 2011 American Institute of Physics. [doi:10.1063/1.3585655]
\end{abstract}

Lithium (Li) dendrite growth on metal Li anodes represents one of the major safety issues that prevented the wide application of rechargeable Li batteries, ${ }^{1-5}$ although there is renewed interest in developing such high energy density batteries (e.g., $1700 \mathrm{~W} \mathrm{~h} \mathrm{~kg}^{-1}$ for the Li-air cells, comparable to that of gasoline ${ }^{6}$ ). The problem of Li dendrite growth is not a serious issue for today's lithium ion batteries (LIBs), in which metallic Li electrode is not present. Recently, however, nanostructured electrodes are intensively tested targeting more applications such as electric vehicles or tools. ${ }^{7,8}$ Advantages by using various nanowires and nanoparticles are emerging in terms of enhancing the energy and/or power density and enabling facile stress release. ${ }^{9-11}$ However, compared to the bulk counterparts, local environment may change significantly around the nanostructured electrodes and particularly, the electrode/electrolyte interface, resulting in unwanted side reactions. ${ }^{8}$

Herein, we present the direct observation of $\mathrm{Li}$ fiber growth from different nanowire anodes during in situ charging nano-LIBs inside a transmission electron microscope (TEM). ${ }^{12}$ The "open cell" nano-LIB was built using an individual nanowire anode (of $\mathrm{Si}$ or $\mathrm{SnO}_{2}$ ), an ionic liquid electrolyte (ILE, LiTFSI/ $\mathrm{P}_{14}$ TFSI, see Ref. 13 for details), and a bulk $\mathrm{LiCoO}_{2}$ cathode (Fig. S1). Long Li fibers were found on the tips of the nanowires after charging, which was attributed to the high electric field at the high-curvature tips induced $\mathrm{Li}^{+}$reduction and $\mathrm{Li}$ plating. Besides, lithium fluoride $(\mathrm{LiF})$ thin film was found on the Li fibers from the electron diffraction patterns (EDPs), evidencing decomposition of the electrolyte and consistent with the observed thick solid electrolyte interface (SEI) layers. Figure 1 shows the Li fiber growth from a $\mathrm{SnO}_{2}$ nanowire anode after charging. Figure 1(a) shows the pristine $\mathrm{SnO}_{2}$ nanowire before charging. The

${ }^{a)}$ Electronic mail: jhuang@sandia.gov. nanowire was straight and clean. After being charged at a constant potential of $-3.8 \mathrm{~V}$ with respect to the $\mathrm{LiCoO}_{2}$ cathode for $50 \mathrm{~min}$, the nanowire elongated and was bent [Fig. 1(b)]. When we pulled the charged nanowire out of the ILE, there was a big chunk of jelled electrolyte adhered to the immersed part of the nanowire. Intriguingly, there was a long tail with light contrast $(\sim 35 \mu \mathrm{m}$ long) attached to the sharp apex of the jelled electrolyte. Figure 1(c) and movie S1 show the pull-out process of the long tail with unique "necklace" (microcrystals decorated fiber) morphology. One of the particles on the necklace is highlighted [Fig. 1(d)], with a well-defined polyhedral shape. There were also some fibers protruding from the thick jelled electrolyte [Fig. 1(e), also marked by the red triangle in Fig. 1(b)]. The electron energy loss spectra (EELS) from these crystals confirmed their main component being metallic lithium [Fig. 1(f)], as multiple plasmon peaks were clearly seen with primary energy loss of $7.2 \mathrm{eV}$, being consistent with the characteristic peaks from pure Li metal. ${ }^{14}$

The Li fiber growth was also observed when Si nanowire anodes were charged (Fig. 2 and Figs. S2-S5). Figure 2(a) shows pristine $\mathrm{Si}$ nanowire with uniform diameter. After charging at $-4 \mathrm{~V}$ against $\mathrm{LiCoO}_{2}$ for $95 \mathrm{~min}$, the nanowire swelled and there was also jelled electrolyte formed on the immersed part of the nanowire [Fig. 2(b)]. A Li fiber, about $1.5 \mu \mathrm{m}$ thick and $7.5 \mu \mathrm{m}$ long, was seen after the nanowire was pulled out of the ILE [Fig. 2(b)]. Again the lithium fiber was grown on the sharp apex at the end of the jelled electrolyte. EDPs from such crystals confirmed that they were single crystalline Li metal (body-centered cubic structure, $a=3.501 \AA$ ) covered with a thin layer of polycrystalline lithium fluoride ( $\mathrm{LiF}$, face-centered cubic structure, $a$ $=4.027 \AA$ ) (Figs. S2 and S3). Because LiF was not present in the original electrolyte, it directly evidenced decomposition of the electrolyte (fluorine from the $-\mathrm{CF}_{3}$ groups of LiTFSI and $\mathrm{P}_{14}$ TFSI molecules). 


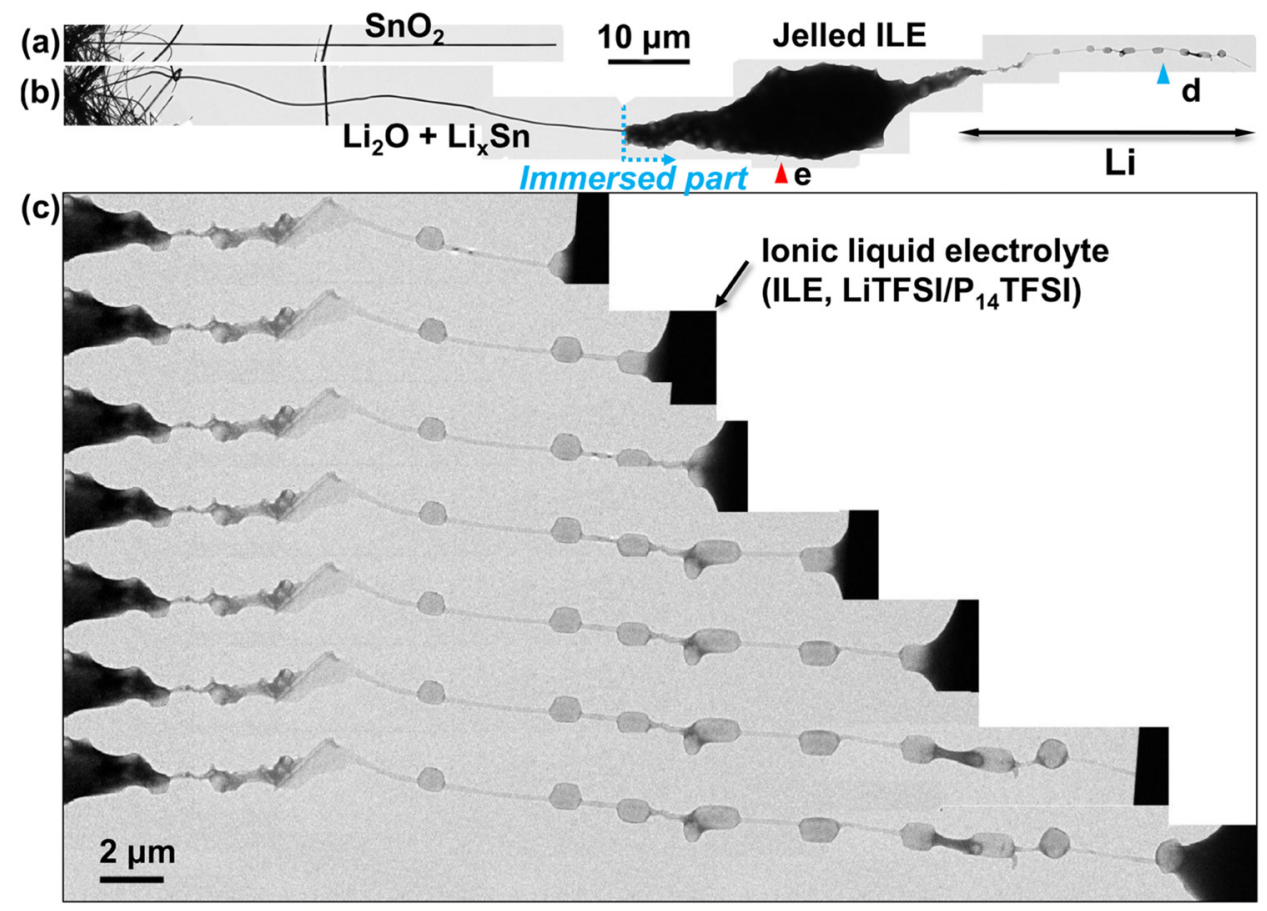

(d)

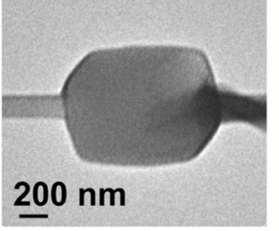

(e)

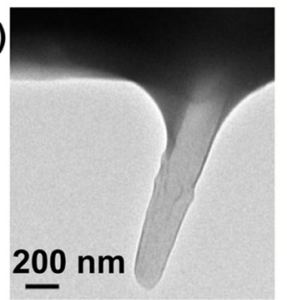

(f)

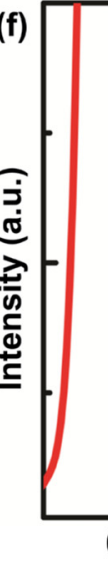

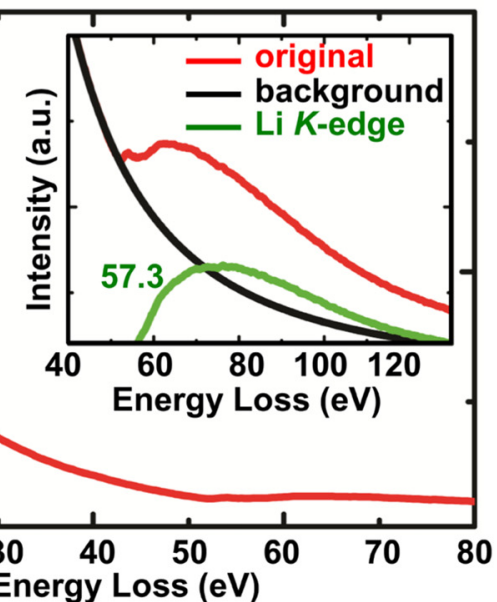

FIG. 1. (Color online) Li necklace formation during charging a $\mathrm{SnO}_{2}$ nanowire. (a) Pristine $\mathrm{SnO}_{2}$ nanowire before charging. (b) Full image of the $\mathrm{SnO}_{2}$ nanowire after charging. The segment above the ILE was bent and elongated, while the segment immersed in the ILE had a large chunk of jelled electrolyte on it. At the end of the jelled electrolyte, there was the long chain of Li necklace. (c) Image series showing the pulling-out process of the Li necklace from the ionic ILE. The Li crystals on the fiber were mostly faceted. (d) Magnified image of the Li crystal marked by the blue triangle in (c). (e) Magnified Li fiber tip protruding out of the thick jelled electrolyte, as marked by the red triangle in (c). (f) EELS from the Li crystals. The multiple plasmon peaks agreed well with those from metallic lithium. The inset shows the Li K-edge with significantly lower intensity than that of the primary plasmon peak, indicating tiny amount of Li compound formed on the Li fiber.
Figures 2(c) and 2(d) illustrate the possible mechanism of $\mathrm{Li}$ fiber growth on a nanowire anode. From the experimental observations, the jelled electrolyte formation occurred on all the surfaces in contact with the electrolyte, however, Li fiber growth took place predominantly on the tip of the nanowires or the jelled electrolyte. Consider the capacitor approximation of the two electrodes separated by an electrolyte, the surface charge density is related to the surface curvature at equilibrium. ${ }^{15,16}$ Due to the largest curvature of the apex, the surface charge density, $\sigma$, should be the highest at the nanowire tip. The electric field at the tip is strongly enhanced, $E=\sigma / \varepsilon$ ( $\varepsilon$ being the dielectric constant of the electrolyte around the nanowire), is thus the highest [Fig. 2(c)]. In the case of a sharp apex against a planar plate with a potential of $V$, the electric field on the sharp apex is strongly enhanced as compared to that of two parallel plates capacitor, in the former, the effective electric field $E$ is inversely proportional to the apex radius $r$, i.e., $E \propto V / r$. As a result of this very high electric field at the tip or so-called "tip effect," ${ }^{, 15-18}$ Li plating took place. As the Li deposition at the tip continues, the high electric field preserves and sustains the continuous $\mathrm{Li}$ growth into a long fiber. Lateral growth is also possible, since charge may be concentrated at local protrusions on the fiber, which accounts for the formation of the necklace-shaped fiber (Fig. 1). Since the electric field is also present on the sidewalls of the jelled electrolyte, it is likely that $\mathrm{Li}$ fiber growth occurs at a much higher threshold of electric field than SEI formation. This may explain that Li fiber growth is not severe in conventional LIBs.

Previous studies have shown strong square $\left(J^{2}\right)$ dependence of Li dendrite growth on current density, $J .^{3}$ Although the actual current was hardly measured in the in situ lithiation of individual nanowires due to their finite mass involved, ${ }^{12}$ the lithiation current $\left(i_{C}\right)$ could be estimated on the order of $\sim 1 \mathrm{pA}$ based on the observed material conversion $\left[\mathrm{M}+x \mathrm{Li}^{+}+x \mathrm{e}^{-} \rightarrow \mathrm{Li}_{x} \mathrm{M}\right.$, Fig. 2(d)]. The current density at the tip was calculated to be $\sim 10^{4} \mu \mathrm{A} \mathrm{cm}^{-2}$ by assuming cross section area of $10^{4} \mathrm{~nm}^{2}$, significantly higher than the typical values in the previous $\mathrm{Li}$ dendrite formation tests, for instance, $\sim 10^{2} \mu \mathrm{A} \mathrm{cm}^{-2}$ for the Li/polymer/Li cells. ${ }^{3} \mathrm{At}$ such highly concentrated electric field and current density, it is not surprising that $\mathrm{Li}$ deposition and electrolyte decomposition would happen. The formation of $\mathrm{Li}$ fiber with the body-centered cubic structure (the room temperature phase) was expected, because the Joule heating effect was negli- 


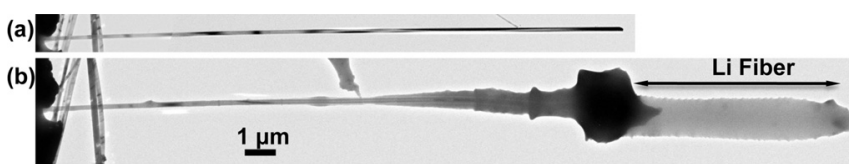

(c)
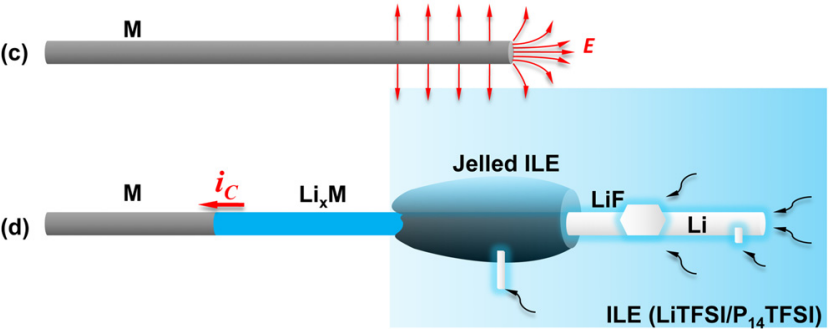

FIG. 2. (Color online) Li fiber formed during charging a Si nanowire. (a) Pristine Si nanowire. (b) Morphology of the whole nanowire after charging. The segment above the ILE swelled and thick jelled electrolyte formed on the immersed segment. A wide and long Li fiber with bright contrast formed on the tip of the nanowire. [(c) and (d)] Schematic illustrations of Li fiber growth on a nanowire anode, $\mathrm{M}$. The dense electric field lines at the apex illustrate the tip effect (c). Li plating takes place on the nanowire tip due to the high electric field, while SEI forms on the whole immersed surfaces at a lower threshold of electric field (d). Lithiation also takes place above the ILE surface with the conversion of $\mathrm{M}$ to $\mathrm{Li}_{\mathrm{x}} \mathrm{M}$.

gible with the bulk electrolyte as the heat sink.

Our finding of the directional growth of long Li fiber in the nano-LIBs has important implications for LIBs using nanowire electrodes. As the Li fiber grows directionally from the nanowire tip to length up to tens of microns, it may short the two electrodes earlier than expected, given the interelectrode distance is greatly reduced in nanobatteries. This brings up precaution about cell safety when nanomaterials are used instead of compact and smooth, bulk electrode materials in LIBs. Structural engineering and surface modification may be necessary to mitigate the adverse effects of using nanomaterials. For example, the dramatic effect on cycling lifetime by coating a very thin alumina layer was demonstrated in particulate $\mathrm{LiCoO}_{2}$ cathode. ${ }^{19}$

In conclusion, we have observed that Li fiber growth can occur in a lithium ion battery where a nanowire anode is used. The fiber grew directionally to a length up to several tens of microns, which was attributed to strong electric field enhancement in the sharp nanowire tip. Electrolyte decomposition was also confirmed by the formation of $\mathrm{LiF}$ and large solid materials deposited on the nanowire. Our results illustrate the importance of basic research on electrochemical processes near nanostructured electrodes, for developing next-generation lithium ion batteries.

Portions of this work was supported by a Laboratory Directed Research and Development (LDRD) project at Sandia National Laboratories (SNL) and partly by the Science of Precision Multifunctional Nanostructures for Electrical Energy Storage (NEES), an Energy Frontier Research Center (EFRC) funded by the U.S. Department of Energy, Office of Science, Office of Basic Energy Sciences under Award No.
DESC0001160. The LDRD supported the development and fabrication of platforms. The NEES center supported the development of TEM techniques, and some of the additional platform development, and fabrication and materials characterization. CINT supported the TEM capability and the fabrication capabilities that were used for the TEM characterization; in addition, this work represents the efforts of several CINT users, primarily those with affiliation external to Sandia National Laboratories. This work was performed, in part, at the Sandia-Los Alamos Center for Integrated Nanotechnologies (CINT), a U.S. Department of Energy, Office of Basic Energy Sciences user facility. Sandia National Laboratories is a multiprogram laboratory operated by Sandia Corporation, a wholly owned subsidiary of Lockheed Martin Co., for the U.S. Department of Energy's National Nuclear Security Administration under Contract No. DE-AC0494AL85000. S.M. would like to acknowledge NSF under Grant No. CMMI 08010934 through University of Pittsburgh and Sandia National Laboratory support. J.L. and A.K. acknowledge support by NSF under Grant Nos. DMR0520020 and DMR-1008104, and AFOSR under Grant No. FA9550-08-1-0325.

${ }^{1}$ F. Orsini, A. Du Pasquier, B. Beaudoin, J. M. Tarascon, M. Trentin, N. Langenhuizen, E. De Beer, and P. Notten, J. Power Sources 76, 19 (1998).

${ }^{2}$ M. Dollé, L. Sannier, B. Beaudoin, M. Trentin, and J. M. Tarascon, Electrochem. Solid-State Lett. 5, A286 (2002).

${ }^{3}$ M. Rosso, C. Brissot, A. Teyssot, M. Dolle, L. Sannier, J. M. Tarascon, R. Bouchetc, and S. Lascaud, Electrochim. Acta 51, 5334 (2006).

${ }^{4}$ R. Bhattacharyya, B. Key, H. L. Chen, A. S. Best, A. F. Hollenkamp, and C. P. Grey, Nature Mater. 9, 504 (2010).

${ }^{5}$ S. Tobishima, Y. Sakurai, and J. Yamaki, J. Power Sources 68, 455 (1997).

${ }^{6}$ G. Girishkumar, B. McCloskey, A. C. Luntz, S. Swanson, and W. Wilcke, J. Phys. Chem. Lett. 1, 2193 (2010).

${ }^{7}$ A. S. Aricò, P. Bruce, B. Scrosati, J. M. Tarascon, and W. Van Schalkwijk, Nature Mater. 4, 366 (2005).

${ }^{8}$ M. Armand and J. M. Tarascon, Nature (London) 451, 652 (2008).

${ }^{9}$ S. H. Ng, J. Z. Wang, D. Wexler, K. Konstantinov, Z. P. Guo, and H. K. Liu, Angew. Chem., Int. Ed. 45, 6896 (2006).

${ }^{10}$ A. Magasinski, P. Dixon, B. Hertzberg, A. Kvit, J. Ayala, and G. Yushin, Nature Mater. 9, 353 (2010).

${ }^{11}$ C. Kim, M. Noh, M. Choi, J. Cho, and B. Park, Chem. Mater. 17, 3297 (2005).

${ }^{12}$ J. Y. Huang, L. Zhong, C. M. Wang, J. P. Sullivan, W. Xu, L. Q. Zhang, S. X. Mao, N. S. Hudak, X. H. Liu, A. Subramanian, H. Y. Fan, L. A. Qi, A. Kushima, and J. Li, Science 330, 1515 (2010).

${ }^{13}$ See supplementary material at http://dx.doi.org/10.1063/1.3585655 for experimental details, Li fibers grown on other Si nanowires after charging, and a movie showing Li necklace being pulled out from the electrolyte.

${ }^{14}$ D. R. Liu and D. B. Williams, Philos. Mag. B 53, L123 (1986).

${ }^{15}$ L. Enze, J. Phys. D: Appl. Phys. 19, 1 (1986).

${ }^{16}$ L. Enze and J. Phys, J. Phys. D: Appl. Phys. 20, 1609 (1987).

${ }^{17}$ R. Xiao, S. Il Cho, R. Liu, and S. B. Lee, J. Am. Chem. Soc. 129, 4483 (2007).

${ }^{18}$ M. J. Siegfried and K. S. Choi, Angew. Chem., Int. Ed. 47, 368 (2008).

${ }^{19}$ Y. S. Jung, A. S. Cavanagh, L. A. Riley, S. H. Kang, A. C. Dillon, M. D. Groner, S. M. George, and S. H. Lee, Adv. Mater. (Weinheim, Ger.) 22, 2172 (2010). 